Article

\title{
Dynamic Inspection Interval Determination for Efficient Distribution Grid Asset-Management
}

\author{
Thorben Neugebauer ${ }^{1}$, Thomas Wolgast ${ }^{2, *}$ and Astrid Nieße ${ }^{2}$ \\ 1 Group Network Operation, Enercity Netz, 30459 Hannover, Germany; thorben.neugebauer@enercity-netz.de \\ 2 Group Energy Informatics, Leibniz University Hannover, 30167 Hannover, Germany; \\ niesse@ei.uni-hannover.de \\ * Correspondence: wolgast@ei.uni-hannover.de; Tel.: +49-511-762-2912
}

Received: 1 July 2020; Accepted: 27 July 2020; Published: 29 July 2020

check for updates

\begin{abstract}
Asset-management accounts for a significant share of grid operators' influenceable costs, which are under high economic pressure due to deregulation. In asset-management, visual inspections are the main measure to determine asset conditions. In the context of digitalization, we propose a concept to acquire additional information about the condition of distribution substations and to derive flexible inspection intervals from this information, in contrast to fixed intervals that are common in scientific literature and practice. Thus, knowledge about the substations increases, inspection intervals can be expanded dynamically, and significant cost savings are possible, which we demonstrate by the example of a German distribution grid operator.
\end{abstract}

Keywords: condition assessment; maintenance; flexible scheduling; digitalization; digitization; substation; economic potential

\section{Introduction}

The deregulation of the energy market intensifies economic pressure on grid operators in Europe and Germany. Maintenance costs of the physical infrastructure represent a significant amount of their influenceable costs. Asset-management was introduced to optimize the long-term compromise between maximum security of supply and minimum maintenance costs. Especially the transition from classic time-based maintenance to modern condition-based maintenance strategy promises considerable savings potential and is investigated extensively [1]. The inspections, however, are mostly assumed to be performed in constant time intervals or not considered at all in asset-management research. Inspections are the main process to evaluate the asset conditions and to determine if and when maintenance measures are required to guarantee security of supply [2]. On the one hand, that makes inspections an important part of modern asset-management, because decisions for condition-based maintenance require a reliable data basis. On the other hand, most inspections do not reveal any deficiencies [1], which implies potential for cost reduction.

For this reason, we examine the possibility that a knowledge-dependent and dynamic inspection process results in significant savings of working hours and therefore costs. The idea is that not only visual inspections can bring knowledge gain about the asset condition, but that the substations are visited frequently for several other reasons. These operations can be used for further and systematic data acquisition. Our hypothesis is that inspection intervals can be dynamized based on these data, which results in:

(a) cost and time savings, if the inspection intervals can be expanded because no further knowledge gain is expected from an inspection,

(b) better reliability, if a critical state is suspected because the necessity for inspection and maintenance can be determined earlier. 
Additionally, expanded inspection intervals reduce the pressure on grid operators to find new electrician specialists, which is an important factor considering the shortage of specialists.

This work focuses on substations in medium voltage (MV) distribution grids and was carried out in cooperation with the enercity Netz company (https:/ / www.enercity-netz.de/index.html; last visit: 7 May 2020), a German distribution system operator (DSO). We consider the regulations and the specific situation in Germany. However, our methodology and ideas are applicable to the general case of asset-management of distribution substations, because inspections to determine maintenance need are common all over the world in asset-management. Therefore, the methodology is usable outside of Germany as well. The contributions of this work are:

1. Options are examined to systematically collect and digitize additional data about the condition of distribution substations with minor additional effort.

2. A procedure is presented to derive dynamic inspection intervals from these data.

3. The economic potential of the approach is calculated based on an exemplary best-case scenario.

The rest of this work is structured as follows: First, we give a short literature overview about asset-management of distribution substations with focus on the inspection process. Second, we introduce enercity Netz, outline the current substation asset-management there, and introduce the used terminology. In Section 4, we work out the requirements that have to be met regarding the inspection procedure of electrical assets. In Section 5, we propose our concept for additional data acquisition and how the inspection intervals can be flexibilized based on these data. Afterward, in Section 6, we calculate the economic potential using a best-case estimation by the example of enercity Netz. Finally, we discuss our results and complete our paper with a conclusion and outlook on further research directions.

\section{Asset-Management of Distribution Substations}

In the context of distribution substation inspection, diverse papers get published that focus on how inspections can be improved, for example by increasing their objectivity [3] or by measuring and reducing their uncertainty [4,5]. However, mostly constant inspection intervals in the range of 1-4 years are assumed [5]. Lacroix et al. [6] present a decision management platform for asset-management considering inspection intervals of five or even ten years. However, only few publications discuss how often the inspection should be performed based on given knowledge about the asset's condition. Dynamic inspection intervals are not in the focus of asset-management research.

Yumbe et al. [7] propose an optimization concept for scheduling inspection intervals of distribution substations, but in the form of an area-based inspection scheme where neighboring assets are inspected together, which is common in Japan. In contrast to the objective of our work, they use the location of the assets as scheduling basis instead of considering the actual condition of the single assets. They further develop that same approach in refs. [8,9]. Xu et al. [10] consider the asset age to derive dynamic inspection rates to minimize total costs, considering costs for maintenance, repair and not supplied energy. Kuntz et al. [11] use a Markov-model to determine the optimal visual inspection frequency of overhead lines in distribution systems considering a trade-off between inspection costs and poor reliability. However, the frequency is calculated only once for a given scenario and the inspection intervals stay constant afterwards, which leaves the asset conditions unconsidered again. Yan et al. [12] also use a Markov-model as a basis to optimize maintenance scheduling, but assume the inspection interval to be periodic and fixed. Bühler and Balzer [13] present a methodology to evaluate the long-term impact of the maintenance activities in the MV grid, which in turn makes optimization possible, but again the inspection interval is assumed to be constant. Schneider et al. [1] give a general overview of asset-management in the energy system. They state that inspection intervals were chosen too much on the safe side in the past. However, they do not propose a solution approach for this problem, thus motivating our research direction.

To sum up, most of the research in the context of asset-management in energy systems either neglects inspections completely or assumes the inspection intervals to be constant. Only the works of 
Yumbe et al. and Xu et al. consider dynamic inspection intervals, but use the location or age of the assets as scheduling basis instead of their respective conditions. In contrast to research in the electric energy system, dynamic inspection intervals are more common in other domains and often used in the context of risk-based inspection. For example, Singh and Markeset [14] propose a concept for risk-based and dynamic inspection scheduling for inspection of oil and gas pipes based on fuzzy logic.

Regarding condition assessment of substations, an alternative approach must be mentioned, too. Instead of regular inspections, on-line monitoring systems can be used to continuously monitor the condition of assets, for example, by measuring partial discharges [15]. However, this work focuses on conventional inspections by specialists.

\section{Status quo at enercity Netz}

enercity Netz is a medium sized urban grid operator of electrical distribution grids in the city of Hannover, northern Germany, which provides electricity for an area of $316 \mathrm{~km}^{2}$ with about 420,000 low voltage (LV) grid connection points (https:/ / www.enercity-netz.de/netze/strom/zahlen-fakten/ index.html; last visit: 7 May 2020). In total, the company operates 2300 distribution substations (https:/ / www.enercity-netz.de/netze/strom/netzgebiet/index.html; last visit: 7 May 2020). A single MV-LV substation has comparatively low investment costs of about $25 \mathrm{k} €$ [16]. Only considering the high number of substations, a total value of $57.5 \mathrm{M} €$ results. On the one hand, that high total number of assets makes asset-management particularly relevant, but also complicates the task, because the comparably low single value of each substation justifies only minimal effort per station.

In the most common version, a distribution substation consists of the building, a MV-switchgear, at least one transformer in the range of 100 to $630 \mathrm{kVA}$, a LV distribution board, diverse measurement devices, and secondary equipment. The distribution substations of enercity Netz have an average age of about 34 years with an expected amortization period and average technical lifetime of 40 years. Thus, most of the assets are at the end of their technical and economical service life. That reflects a germanand world-wide problem of aging assets in the electrical grids $[2,17]$.

In the following, the current asset-management at enercity Netz is described briefly. To clarify the terminology, the various involved processes are listed and described comprehensively in Table 1.

Table 1. Overview of substation asset management nomenclature (For definitions refer to norm [18]).

\begin{tabular}{ll}
\hline Process & Description \\
\hline $\begin{array}{ll}\text { (Visual) Inspection } \\
\text { Maintenance }\end{array}$ & $\begin{array}{l}\text { Regular visual check of the asset condition. } \\
\text { All measures to physically alter the asset's state (performed depending on } \\
\text { inspection results). }\end{array}$ \\
Revision & Inspection, including function check, and immediate maintenance, if necessary. \\
Visit & All non-maintenance related tasks within a substation (e.g. switching actions). \\
Station control & Generic term for all operations within a substation (Inspection, maintenance, \\
operation & revision, visit). \\
Task & Task within the workforce management system to schedule a station control \\
& operation.
\end{tabular}

A visual inspection is performed at fixed time intervals, with a maximum interval of four years [19]. In practice, the actual time-frame is usually in the range of about one to two years. Inspection means all operations to determine the asset condition, for example purely visual inspections or function checks. However, in the context of this work, the term inspection always refers to visual inspection. The inspection is performed by substation foremen by filling out a pre-defined checklist in a mobile end device. The results of the inspection are collected and used to calculate a single metric that describes the asset condition. This metric lays the basis for the decision if and when maintenance measures are performed. Maintenance includes all actions to slow down the degradation process 
of the assets, for example corrosion protection measures or tightening of screw connections.After maintenance, the asset condition can be assumed to be good again. Besides the inspection dependent maintenance, there is a second maintenance process running parallel; the revision restores the asset condition to a very good state by performing an extended inspection - including function checks and subsequent repairs, if necessary. Generally, revision is performed in larger time intervals than the inspection, based on recommendations of the asset manufacturers. Additionally, the substation foremen visit the substations at irregular intervals to perform non-maintenance related actions, for example switching operations. These activities are called visits in the following. Currently, the information about the asset condition gained at these visits are not documented in any way and therefore not utilized for asset-management either. Only if the substation is in extremely deficient condition during visit, measures are taken. The term station control operation is utilized here as the generic term for all activities within a substation and thus includes inspections, revisions, maintenance actions, and visits. All these processes are coordinated by a workforce management (WFM) system that generates work tasks for the substation foremen. For our work, we assume that all these tasks get executed by electrical specialists, which is the case for enercity Netz and can be assumed for grid operators worldwide.

\section{Requirements Analysis}

In Table 2, all requirements are listed that have to be considered regarding the inspection process.

Table 2. Requirements regarding the inspection process and data acquisition.

\begin{tabular}{lll}
\hline & Requirement & Necessity \\
\hline R1 & Fulfillment of safety obligation & Must \\
R2 & Compliance with norms and standards & Must \\
R3 & Digital data acquisition & Should \\
R4 & High usability for the workers & Should \\
R5 & Minimal time expenditure & Should \\
R6 & Hard-facts preferred over soft-facts & Should \\
\hline
\end{tabular}

Especially, some hard requirements have to be met, considering laws and standards. First, the legal duty to maintain safety with respect to the premises and electrical equipment must be fulfilled under all circumstances. It is called safety obligation (requirement R1). The German Bundesgerichtshof (federal supreme court) describes the safety obligation in a judgement of 2012 as follows:

The person who creates or maintains a source of danger has the duty to take the necessary and reasonable precautions to prevent damage to others. Translated from the German original judgment: "Derjenige, der eine Gefahrenquelle schafft oder unterhält, hat die Pflicht die notwendigen und zumutbaren Vorkehrungen zu treffen, um Schäden anderer zu verhindern" [20].

Examples for compliance with the safety obligation in the context of substation asset-management are compliance with fire regulations or guaranteeing that the electrical installations are not accessible for external parties. The specifications regarding safety obligation in electrical assets are described in the DIN V VDE 0109-2 [21]. The safety obligation is fulfilled, if regular inspections prove that an electrical system complies with all necessary safety regulations and standards.

Second, the conduct of the inspection process has to comply with the DIN VDE 0105-100 [22] (R2). Either the inspections have to be performed regularly or continuous monitoring is possible, alternatively. This work focuses on regular inspections only. The time interval between the inspections is not strictly defined, but the standard dictates that it must be chosen by the grid operator on basis of (a) the asset type, (b) its application and operation, (c) quantity and quality of maintenance, and (d) external influences. The scale of inspection intervals may span over up to four years [19]. 
Further, some soft requirements should be considered, too. First, all data should be collected digitized and systematically to ensure that these data are available for decision making and scheduling, thus paving the way to further optimization based on digitalization like fully data-driven asset management (R3). Second, a smooth introduction of the system is to be aimed at. All adjustments in the process must be made under the objective of high usability for the workers (R4). Especially a minimal time expenditure for the data acquisition is aspired, not only because of usability, but also to ensure that additional costs and working hours do not surpass the cost savings (R5) [5]. That is particularly important in the distribution grids where a high total number of inspections are performed, single assets have only low value, and relatively low follow-up costs arise in the case of an outage. Finally, even after visual inspection, a residual uncertainty about the asset's condition remains, because the inspection process itself is error-prone and often subjective [4]. Especially soft-facts that cannot be measured objectively, but are acquired based on the impression of the substation foreman, are highly subjective and therefore dependent on several factors like the experience, expertise, mood, expectations, and willingness to take risk of the worker, or external conditions (e.g. weather). Consequently, measurable hard-facts about the asset conditions should be acquired preferably (R6) [3].

\section{Dynamic Inspection Intervals}

In the following, we present our approach and framework for dynamic inspection intervals. The conventional inspection process (identified with superscript $i$ ) is performed in fixed time intervals $z$, which is visualized in Figure 1. The interval $z$ is chosen by the grid operator based on legal requirements and risk-tolerance.

$$
\tau_{n+1}^{\mathrm{i}}=\tau_{n}^{\mathrm{i}}+z
$$

To allow for dynamic inspection intervals, Equation (1) is modified to (2):

$$
\tau_{n+1}^{\mathrm{i} *}=\tau_{n}^{\mathrm{i}}+z_{n}
$$

The asterisk indicates a shifted inspection date. The difference between the dynamic interval $z_{n}$ and the fixed interval $z$ results in the time savings potential which allows to calculate the economic impact of the suggested concept using the hourly labour costs. The dynamic inspection process is exemplarily shown in Figure 2.

$$
\Delta z_{n}=z_{n}-z
$$

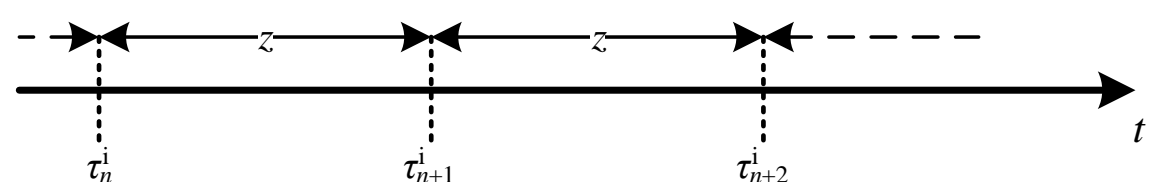

Figure 1. Classic inspection process with temporally constant intervals.

As additional data sources to calculate the dynamic inspection date, the revision and the substation visits are taken into account. That allows to postpone the inspection task, if the asset condition is already sufficiently well-known, or to perform inspection earlier, if a critical condition is suspected. If no revision or visit took place, the standard interval $z$ is used furthermore. The suggested procedure to calculate such dynamic inspection intervals is described in the following. Here, it is important to mention that this work only shows the general idea of dynamic inspection interval determination in an exemplary way. The practical implementation of such a procedure is highly dependent on the grid operator company and the number and types of assets in the respective grid. 


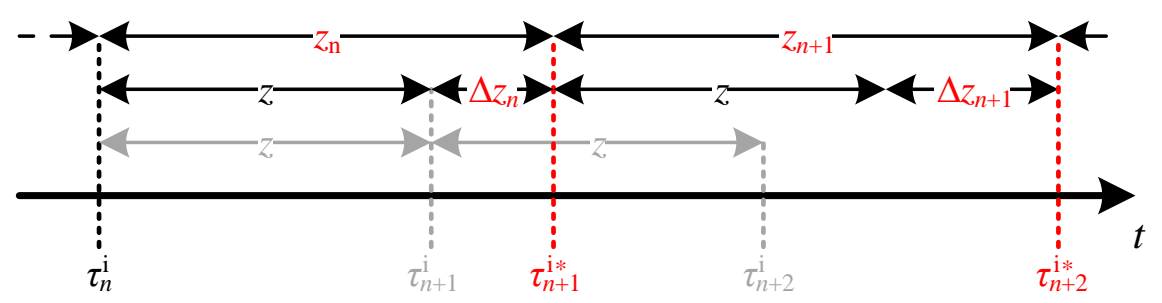

Figure 2. Proposed inspection process with dynamic inspection intervals.

\subsection{Revision}

Revision is performed in fixed time intervals of several years. The cycle is usually longer than the inspection cycle, which implies that a maximum of one revision is possible between two inspections. The acquired information can be utilized for postponing the next inspection. During a revision, a visual inspection, function checks and corrections of deficiencies are performed. Consequently, the asset condition as well as the knowledge about it can be assumed to be optimal after revision, which makes an inspection directly afterwards unnecessary. However, the revision (superscript $r$ ) must fall between two inspection dates to be considered. Therefore, the following condition must be true:

$$
\tau_{n}^{\mathrm{i}}<\tau^{\mathrm{r}}<\tau_{n+1}^{\mathrm{i} *}
$$

Regarding the interval extension, the safety obligation (superscript s) must be considered. The grid operator defines a maximum time-interval $z^{\mathrm{s}}$ after which the compliance with the safety obligation must be checked again. In this work, we assume the interval for safety obligation and inspection to be the same, because the inspection is the main instrument to ensure compliance with the safety obligation.

$$
z^{\mathrm{s}}=z^{\mathrm{i}}=z
$$

The difference between revision date and postponed inspection date must not surpass the maximum interval of the safety obligation:

$$
\tau_{n+1}^{\mathrm{i} *}-\tau^{\mathrm{r}} \stackrel{!}{\leq} z
$$

Consequently, the maximum valid cycle extension by revision consideration is as follows:

$$
\begin{gathered}
\tau_{n+1}^{\mathrm{i} *}=\tau^{\mathrm{r}}+z=\tau_{n}^{\mathrm{i}}+z_{n} \\
\Rightarrow z_{n}=\tau^{\mathrm{r}}-\tau_{n}^{\mathrm{i}}+z \\
\Rightarrow \Delta z_{n}^{\mathrm{r}}=\tau^{\mathrm{r}}-\tau_{n}^{\mathrm{i}}=z_{n}^{\mathrm{ir}}
\end{gathered}
$$

In conclusion, the inspection interval can be enlarged by the time interval between the previous inspection date $\tau_{n}^{\mathrm{i}}$ and the revision date $\tau^{\mathrm{r}}$, as visualized in Figure 3 .

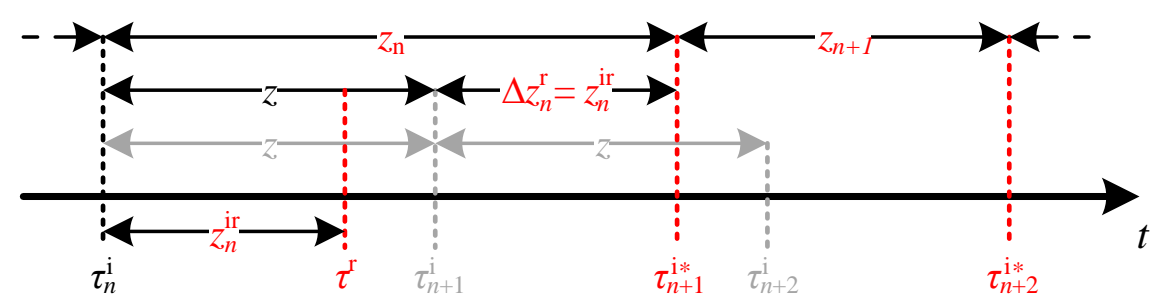

Figure 3. Dynamic inspection intervals considering revision. 


\subsection{Non-Maintenance Related Visits}

Compared to revisions, the visits do not effectuate changes in the asset conditions. Also, the visits are not conducted time- or condition-related, but depending on the tasks in the electricity grid. Therefore, visits can happen randomly any number of times within a given inspection interval. In one interval multiple visits may take place, while in another interval not a single visit happens. Consequently, the visits are identified by an additional index $m$ to differentiate between multiple visits within the same interval.

The objective is to acquire the specialist know-how of the substation foremen, who visit the substations frequently anyhow, to gain further knowledge about their respective condition, which can be fed into a digitized process. For that reason, a short assessment of the asset conditions shall be given after every visit of a specialist that is sufficiently qualified to do so. In the process, the operational execution gets interfered with, which is why high usability requirements must be met here (R4 \& R5).

To achieve simple usability with high expressiveness, we propose a straightforward four-stage rating scale where 1 is the best and 4 is the worst rating of the overall impression, see Figure 4 . An even number of stages is chosen to force the user to decide between an overall negative or positive impression of the asset conditions. As scale point designation, a combination of visual, verbal, and numerical rating points is chosen. The visual emojis generate an easy and clear assessment option. The verbal explanation and the numerical rating improve objectivity and the differentiation of the stages from each other.

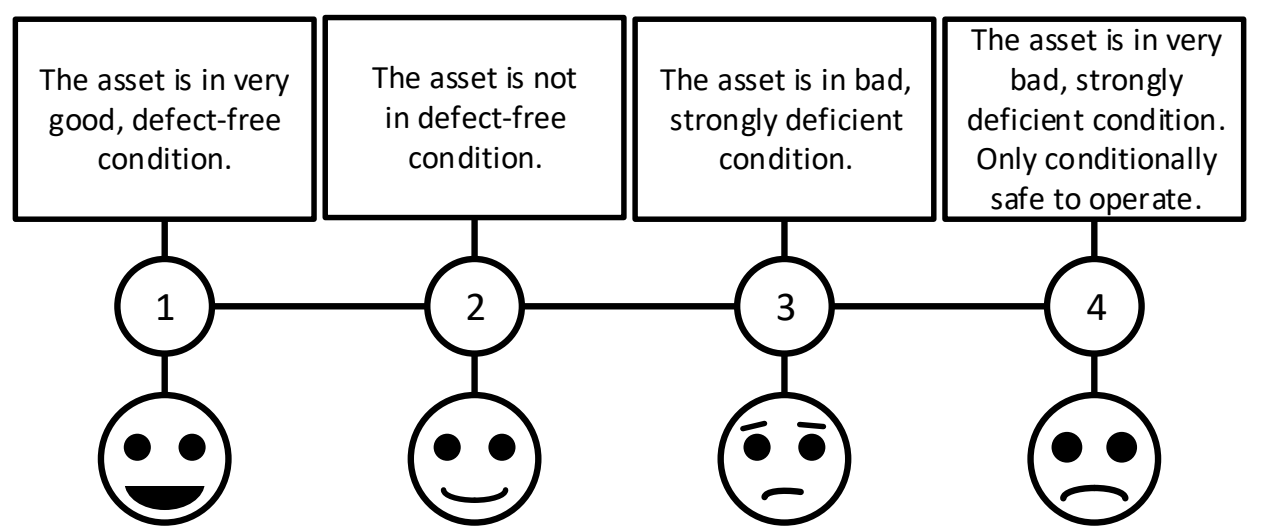

Figure 4. Simple four-stage rating scale for the assessment of substation conditions. Remark: The internal understanding at enercity Netz is that "not defect-free" is synonymous with sufficient asset condition and therefore seen as positive.

Analogous to the revision process, we present a model for the consideration of non-maintenance substation visits. Again, the visits (superscript $v$ ) are only relevant for dynamization, if a visit $m$ falls between two inspection dates, which is verified by the following condition:

$$
\tau_{n}^{\mathrm{i}}<\tau_{m}^{\mathrm{v}}<\tau_{n+1}^{\mathrm{i} *}
$$

If the condition is false and no visit took place in the considered time-frame, no adjustment of the inspection interval based on visit data is done, because no further knowledge is gained. The fulfillment of the safety obligation is considered as follows:

$$
\tau_{n+1}^{\mathrm{i} *}-\tau_{m}^{\mathrm{v}} \stackrel{!}{\leq} z
$$

However, regarding the visits, the safety obligation is not the limiting factor, but the ensuring of the asset functionality. A visit only provides a subjective condition assessment and neither an exact condition determination nor a physical adjustment of the asset condition. Analogous to the visual inspection, the knowledge about the asset condition increases with the number and accuracy 
of assessments. Thereby, not only the absolute number of visits is essential, but also their temporal distribution. For example, five visits evenly distributed between two inspections provide higher benefit compared to five visits in a single week. The same applies, if the visit takes place directly after a revision, maintenance action, or visual inspection. To consider this problem, a minimal time gap is defined between a visit and any other operation. A visit must take place at least $\Delta \tau_{\min }^{\mathrm{v}}$ after the last operation to be considered for dynamization of the inspection, which is fulfilled if the following conditions are true:

$$
\begin{gathered}
\tau_{m}^{\mathrm{v}}-\tau_{n}^{\mathrm{i}} \geq \Delta \tau_{\min }^{\mathrm{v}} \\
\tau_{m}^{\mathrm{v}}-\tau_{m-1}^{\mathrm{v}} \geq \Delta \tau_{\min }^{\mathrm{v}} \\
\tau_{m}^{\mathrm{v}}-\tau^{\mathrm{r}} \geq \Delta \tau_{\min }^{\mathrm{v}}
\end{gathered}
$$

Regarding the interpretation of the assessment (see Figure 4), a case distinction is done: If the rating was extremely poor (rating 4) and the assessment hints to reduced functionality, the WFM-system generates a task for visual inspection at that station immediately, because security of supply may be endangered. For all other ratings, the following procedure is implemented: Visits that were rated with 1 or 2 increase the number of positive ratings $V_{n}^{\text {pos }}$ by one, while all visits rated with 3 increase the number of negative ratings $V_{n}^{\text {neg }}$ by one. To prevent that the interval increases too much and to make sure that inspections are still executed, the possible enlargement rate of the inspection interval decreases with increasing number of assessments. That is described by the following equation:

$$
\Delta z_{n}^{\mathrm{v}}=\sum_{j=1}^{V_{n}^{\text {pos }}} \frac{\Delta \tau_{\min }^{\mathrm{v}}}{j}-\sum_{j=1}^{V_{n}^{\mathrm{neg}}} \frac{\Delta \tau_{\min }^{\mathrm{v}}}{j}
$$

Corresponding to (15), the inspection interval increases with the number of positive ratings and decreases with the number of negative ratings. Both $V_{n}^{\text {pos }}$ and $V_{n}^{\text {neg }}$ are reset to zero after inspection or revision. The minimal time gap $\Delta \tau_{\min }^{\mathrm{v}}$ is the maximum interval extension that can result from a single visit, to always guarantee compliance with the safety obligation. Figure 5 shows an exemplary interval extension. In the first inspection interval, three visits take place with positive ratings each. These are included in the calculation of the inspection date $\tau_{n+1}^{\mathrm{i} *}$.

Regarding the usage of substation visits, it is important to mention that all maintenance actions can be categorized as visits, as well, because substation foremen visit the substation and are able to gain an impression of the asset's overall condition.

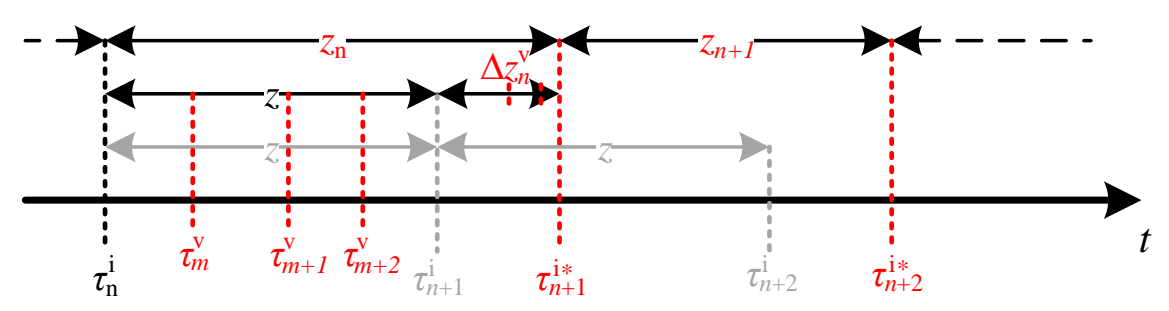

Figure 5. Dynamic inspection process considering the substation visits.

\section{Economic Potential}

In the previous section, we proposed a concept on how to calculate dynamic inspection intervals by integrating existing information sources. Now, we calculate the economic potential of the dynamic inspection intervals with best-case estimations for reduction of work hours by the example of enercity Netz.

The best-case estimation is performed in relative terms compared to an assumed inspection interval $z$ of 1.5 years.

$$
z=1.5 y
$$


For the revision, we assume a fixed interval of eight years.

$$
z^{r}=8 y
$$

Regarding visits, Equations (12) to (14) introduced $\Delta \tau_{\min }^{\mathrm{v}}$ as the minimal time gap between visit and last station control operation to be considered. Exemplarily, that minimal gap is chosen as $z / 6$ here (3 months):

$$
\Delta \tau_{\min }^{\mathrm{v}}=z / 6
$$

In the following, we calculate the average interval extension $\overline{\Delta z}$ for the best-case scenarios. That allows us to calculate the expected relative working hours savings potential $P(\overline{\Delta z})$ of the dynamic inspection intervals:

$$
P(\overline{\Delta z})=\frac{\overline{\Delta z}}{z+\overline{\Delta z}}
$$

For one inspection task, we assume an average duration of 150 minutes, which is realistic for enercity Netz, and labor costs of $60 € / \mathrm{h}$ [3]. Applied to enercity Netz, this results in about $3800 \mathrm{~h}$ spent for the inspection of distribution substations every year (see Section 3). Therefore, we estimate the total inspection costs as $230 \mathrm{k} €$ per year. These costs are used as a reference to calculate cost savings potential in the following. First of all, revision and visits are examined independently from each other. Afterward, it is investigated what effects result from the combination of both.

\subsection{Revision}

For cost savings potential, a simple best-case estimation can be done. In the ideal case, the revision date falls exactly on the inspection date, which makes obsolete one inspection every eight years.

$$
\overline{\Delta z^{\mathrm{r}}}=z^{*}-z=\frac{z^{\mathrm{r}}}{\frac{z^{\mathrm{r}}}{z}-1}-z=0.346 \mathrm{y}
$$

On average, $\frac{z^{\mathrm{r}}}{z}=5 . \overline{33}$ inspections take place within an eight-year interval, which gets reduced by one. That is equivalent to an average interval extension $\overline{\Delta z^{\mathrm{r}}}$ of 0.346 years. Using Equation (19), that makes a cost savings potential of $18.74 \%$ by the revision process alone.

\subsection{Visits}

Considering the savings potential of visits, an additional effect must be taken into account, which was irrelevant concerning revision. If a substation visit falls between two inspections and fulfills all the requirements, the inspection interval increases. That, in turn, increases the probability that another visit falls in the new interval and so on. This effect did not need to be considered regarding revision, because a maximum of one revision can take place within an inspection interval. Regarding the visits, normally a maximum of six relevant visits within the inspection interval can be expected, considering the minimal time gap $\Delta \tau_{\min }^{\mathrm{v}}$ of $z / 6$. Taking into account the described effect, the new $\overline{\Delta z^{\mathrm{v}}}$ can be calculated with the following Equation (21), which is based on (15) assuming one visit exactly every three months with positive rating each, which is the best-case again.

$$
\begin{array}{r}
\overline{\Delta z^{\mathrm{v}}}=\sum_{j=1}^{V_{n}^{\mathrm{pos}}} \frac{\Delta \tau_{\min }^{\mathrm{v}}}{j}=0.679 \mathrm{y} \\
\text { with } \quad V_{n}^{\mathrm{pos}}=\left\lfloor\frac{\left(z+\overline{\Delta z^{\mathrm{v}}}\right)}{\Delta \tau_{\min }^{\mathrm{v}}}\right\rfloor=8
\end{array}
$$


Rounding off is required, because the inspection interval must be extended by at least 3 months already so that another visit can be considered. The equation was solved iteratively and results in an average interval extension of 0.679 years, including a maximum of eight relevant substation visits in that interval. Using Equation (19), that results in a total best-case savings potential of $31.16 \%$ with the visits alone.

A clarifying remark concerning this evaluation: The best-case scenario may seem unrealistic, but in 2018 about 8500 visits from personal qualified for inspection took place at enercity Netz. On average, that makes about four operations per station and year, which is almost equivalent to the considered best-case.

\subsection{Combination of Revision and Visits}

The previous two paragraphs demonstrated the cost savings potential of the revisions and the substation visits. Now, we investigate how a superposition of these two factors affects the cost savings potential.

The same procedure as for the revision process alone is executed, see Equation (20). However, this time, instead of the constant inspection interval $z$, the dynamic inspection interval $z_{n}^{\mathrm{v} *}$ resulting

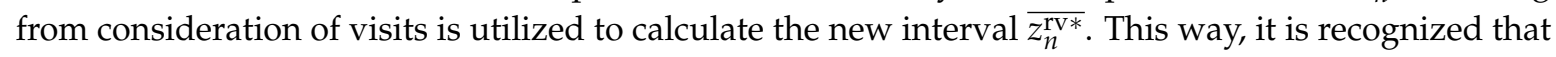
a longer inspection interval gets obsolete by the revision in this case.

$$
\overline{\Delta z^{\mathrm{rv}}}=\overline{z_{n}^{\mathrm{rv} *}}-z=\frac{z^{\mathrm{r}}}{\frac{z^{\mathrm{r}}}{z+\overline{\Delta z^{\mathrm{v}}}}-1}-z=1.495 \mathrm{y}
$$

In total, the inspection period can almost be doubled, which is more than the sum of both single factors revision and visits alone. The superimposed interval extension results in a total cost savings potential of $49.92 \%$ under the assumptions made. That is the sum of both previously calculated relative cost savings potentials with a minor rounding error.

In absolute numbers, cost savings for enercity Netz of $115 \mathrm{k} €$ per year are possible, using the estimated inspection costs as a basis. The new average inspection interval would be almost three years.

\section{Discussion}

We presented a concept to gain additional information about substation conditions and to derive dynamic inspection intervals from this information. The economic potential of the approach was calculated by using a best-case estimation. Now, we discuss our hypothesis that dynamic time intervals for the inspection procedure, based on expanded data acquisition and usage, result in significant cost savings for the grid operator-an idea that has received little attention in literature yet. Also, we critically analyze our approach and point out possible drawbacks of the presented concept. Further, we review if the requirements discussed in Section 4 are fulfilled.

\subsection{Critical Analysis}

In Section 6, we showed that our concept results in possible cost savings of about $50 \%$ of the total inspection costs. That validates the initial hypothesis. In general, the total economic potential increases with the number of assets, which makes the concept more interesting the larger the number of assets is. However, it is important to remember that the best-case potential for savings was estimated and only for an exemplary case. The actual cost savings that would result from practical implementation remain unknown. These are highly dependent on the actual processes within the company and therefore only determinable, if the proposed information acquisition concept gets implemented and evaluated regarding the actual cost savings. On the other hand, it was stated before that inspections only rarely result in actual error detection. That hints that the real-case savings are in the same general range as the best-case potential. 
A similar case applies for the chosen parameters of the concept. For example, the interval extension of the substation visits was chosen arbitrarily to exemplify the concept. These parameters cannot be derived on a theoretical basis alone. Instead, they must be chosen based on the grid operator's respective tasks, difficulties, focal points, and gathered data, as well as willingness to take risks.

Especially the inclusion of the substation visits results in great savings potential, but on the other hand, it is unknown how much time the condition assessment would take in practical use and how that affects the overall cost balance of the concept. In practical implementation, the assessment has to be executed as fast and simple as possible so that the additional expenditure of time does not surpass the time savings.

This work focuses on the cost savings potential of the approach, but neglects the consequences for security of supply. Asset-management is the compromise between costs and security which is why the effects on asset conditions and supply of security should be considered, too. Again, an estimation is hardly possible on a theoretical basis only, but requires test phases. Will the security of supply increase, because of further and more systematic data usage? Or will it decrease, because of a smaller number of inspections?

One contribution of the proposed concept is the additional data acquisition about the asset conditions. This way, not only cost savings are possible, but the data can be used for diverse other purposes, for example the development of asset aging models or the training and usage of machine learning algorithms. However, it must be stated that this relies on the assumption that all deficiencies are to be found. If that is not given, security of supply is threatened. A certain redundancy regarding the inspections - which is the status quo - may be the best overall solution still.

\subsection{Requirement Fulfillment}

In Section 4, we elaborated several requirements that must or should be fulfilled concerning the inspection process (see Table 2). The fulfillment of the safety obligation (R1) was set as a hard constraint for all considered processes and can be seen as fully complied with by the chosen constraints. The norms and standards (R2) mainly specify the compliance with the safety obligation, which is fulfilled with the previous point. Apart from that, they only loosely specify the execution rhythm of the inspections. Inspections are still performed regularly and the new inspection interval is still smaller than the maximum interval of four years. The next requirements are digital data acquisition (R3), high usability (R4), and minimal time requirement (R5) for the substation foremen.Regarding the revision, all these requirements are fulfilled, because the data are already in the WFM system, and there is no workflow change for the workers at all. Only the dynamic determination of the inspection dates must be implemented internally in the WFM system. The situation is different for the visits. Here, all three factors are dependent on the actual practical implementation of the process and cannot be evaluated on a theoretical basis. The last requirement was to replace soft facts by hard facts (R6). Especially regarding the assessment after substation visits, that requirement is not fulfilled, because the assessment is based on the worker's impression of the asset condition, which makes it highly subjective.

Altogether, the requirements are fulfilled for the most part or dependent on the actual implementation of the concept. Only the absence of hard facts about the asset conditions is problematic, but that is a general problem that concerns the conventional visual inspections, too. In ref. [4], Beerboom et al. present and discuss several approaches to reduce subjectivity, for example staff training focused on asset condition assessment.

\section{Conclusions and Outlook}

We presented a concept to acquire additional information about the asset conditions in distribution substations as well as an approach to consider this information for the generation of dynamic inspection intervals that depend upon the asset conditions and the knowledge about it. Further, we estimated the economic potential of the proposed dynamic inspections based on a best-case assessment and discussed the results as well as the proposed concept itself. 
We found that new information sources can be accessed, which were omitted at enercity Netz and in literature until now. Consideration of the revision as inspection replacement allows omitting one inspection every eight years in our exemplary case. And the frequent substation visits that take place anyway allow a further utilization of the substation foremen's know-how that was neglected before. Considering these two aspects, an exemplary best-case estimation of possible cost savings at enercity $\mathrm{Netz}$ was executed. Among the assumptions made and how the parameters were chosen, an average inspection interval doubling resulted. That is equivalent to inspection cost savings of about $50 \%$. This paper demonstrates how a broader data utilization and digitization can lead to significant potential for cost savings. The practical implementation of the concept is currently being discussed at enercity Netz.

The discussion in Section 7 demonstrated that practical data would be required to obtain further information about the potential of the approach. For further research, the data acquisition approach with substation visits should be put into practice. That would allow to determine factual possible cost savings and also to assess the potential impacts on deficiency detection. For example, do the information from visit assessment hint to deficiencies that get found at the inspection later? This way, conclusions can be drawn if the information from substation visits enable a partial replacement of the inspections. Further information gain about the cost savings and the effects on asset conditions may also allow integrating the concept into an optimization algorithm to not only extend the inspection intervals, but to find the optimal inspection dates continuously. In refs. [4,5], Beerboom and Johae discuss techniques how to use uncertainty theory to describe the uncertainty about the assets' conditions. That approach might allow assessing how visits and inspection affect the uncertainty of assets' conditions and how the process can be optimized.

Author Contributions: Conceptualization, T.N. and A.N.; methodology, T.N., T.W. and A.N.; validation, T.N. and T.W.; formal analysis, T.N. and T.W.; investigation, T.N. and T.W.; resources, A.N.; writing- original draft preparation, T.W.; writing-review and editing, T.N., T.W. and A.N.; visualization, T.N. and T.W.; supervision, A.N.; project administration, A.N. All authors have read and agreed to the published version of the manuscript.

Funding: This research received no external funding.

Acknowledgments: This work was done in cooperation with enercity Netz. In particular, we would like to thank Marco Klatt and Sebastian Kerkow from enercity Netz for helpful discussions and valuable comments. Further, we would like to thank Matin Obaidi for his helpful proofreading.

Conflicts of Interest: The authors declare no conflict of interest.

\section{References and Notes}

1. Schneider, J.; Gaul, A.J.; Neumann, C.; Hogräfer, J.; Wellßow, W.; Schwan, M.; Schnettler, A. Asset Management Techniques. Int. J. Electr. Power Energy Syst. 2006, 28, 643-654. [CrossRef]

2. Wan, S. Asset Performance Management for Power Grids. Energy Procedia 2017, 143, 611-616. [CrossRef]

3. Köhn, P.; Schnettler, A.; Schultze, N. Simulation and analysis of assessment procedures for condition based maintenance of MV/LV substations. In CIRED 2016 Workshop; IET: Stevenage, UK, 2017. [CrossRef]

4. Beerboom, D.; Johae, C.; Zdrallek, M.; Schultze, N.; Timmreck, R. Condition assessment of distribution grids using uncertainty theory. In Proceedings of the 23rd International Conference on Electricity Distribution (CIRED 2015), Lyon, France, 15-18 June 2015.

5. Johae, C.; Beerboom, D.; Pawlowski, E.; Zdrallek, M.; Schultze, N.; Timmreck, R. Economical and practicable condition assessment of MV- and LV-distribution grids. CIRED—Open Access Proc. J. 2017, 2017, 128-131. [CrossRef]

6. Lacroix, T.; de Lumley, T.; Pinto, O.; Blonbou, F. Nexans Strategic Asset Management Solution (SAMS): The powerful decison making platform dedicated to DSOs. In Proceedings of the 25th International Conference on Electricity Distribution (CIRED 2019), Madrid, Spain, 3-6 June 2019. [CrossRef]

7. Yumbe, Y.; Hasegawa, T. Inspection Schedule Optimization Technique for Power Distribution Facilities. In Proceedings of the IEEE PES T\&D 2012, Orlando, Florida, USA, 7-10 May 2012.

8. Yumbe, Y.; Hasegawa, T.; Furukawa, N. Optimization Method for Inspection Scheduling of Power Distribution Facilities. IEEE Trans. Power Deliv. 2013, 28, 1558-1565. [CrossRef] 
9. Yumbe, Y.; Miyakoshi, M.; Kondo, M.; Arao, T.; Furukawa, N. Evaluation of Optimization Method for Inspection Scheduling of Power Distribution Facilities Using Maintenance Data Accumulated by Power Utility. IEEE Trans. Power Deliv. 2017, 32, 696-702. [CrossRef]

10. Xu, B.; Xu, S.; Zhang, Y. Determining Optimal Inspection Rates of Power Equipment Considering Opportunistic Maintenance Strategy; ICGEA 2019; IEEE Press: Piscataway, NJ, USA, 2019; pp. 51-55. [CrossRef]

11. Kuntz, P.A.; Christie, R.D.; Venkata, S.S. A Reliability Centered Optimal Visual Inspection Model for Distribution Feeders. IEEE Trans. Power Deliv. 2001, 16, 718-723. [CrossRef]

12. Yang, F.; Kwan, C.M.; Chang, C.S. Multiobjective Evolutionary Optimization of Substation Maintenance Using Decision-Varying Markov Model. IEEE Trans. Power Syst. 2008, 23, 1328-1335. [CrossRef]

13. Buhler, J.; Balzer, G. Long-Term Impact Evaluation of Maintenance Activities on MV/LV Substations. In 2011 IEEE Trondheim PowerTech; IEEE: Trondheim, Norway, 2011; pp. 1-8. [CrossRef]

14. Singh, M.; Markeset, T. A methodology for risk-based inspection planning of oil and gas pipes based on fuzzy logic framework. Eng. Fail. Anal. 2009, 16, 2098-2113. [CrossRef]

15. Caprara, A.; Ciotti, G. On-line PD monitoring of Medium Voltage assets: an innovative approach to improve asset management. In Proceedings of the 25th International Conference on Electricity Distribution (CIRED 2019), Madrid, Spain, 3-6 June 2019. [CrossRef]

16. Johae, C. Realitätsgerechte Zustandsbewertung von Mittelspannungsanlagen durch Einsatz Geeigneter Messverfahren. Ph.D. Thesis, Universität Wuppertal, Wuppertal, Germany, July 2018.

17. Deutsche Energie-Agentur (dena). Ausbau- und Innovationsbedarf der Stromverteilnetze in Deutschland bis 2030; 2012.

18. DIN EN 13306:2018-02: Maintenance-Maintenance Terminology; Trilingual Version EN 13306:2017, 02/2018.

19. Berufsgenossenschaft für Gesundheitsdienst und Wohlfahrtspflege (BGW). DGUV Vorschrift 3: Elektrische Anlagen und Betriebsmittel.

20. VI. Zivilsenat des Bundesgerichtshofs BGH (VI. Civil Senate of the Federal Supreme Court). Judgement of 2012.10.02-VI ZR 311/11 2012.

21. DIN V VDE 0109-2 (VDE V 0109-2):2014-09: Maintenance of Installations and Equipment of Electrical Energy Supply Networks-Part 2: Determination of the Condition of Equipment/Installation (Pre-standard), September 2014.

22. DIN VDE 0105-100 (VDE 0105-100):2015-10: Operation of Electrical Installations-Part 100: General Requirements, 2015. 